\title{
Pulmonary Surfactant Protein D Enhances Ovalbumin Clearance by Aggregations
}

\author{
C.-L. Na, J. Nadesalingam, P. Djiadeu, and N. Palaniyar \\ Lung Biology Research Program, Hospital for Sick Children, Toronto, ON M5G 1X8, Canada
}

Pulmonary surfactant protein D (SP-D) is an innate immune collectin (collagenous lectin) secreted by alveolar type 2 cells and Clara cells in the lungs. Recent studies suggest that SP-D is not only important for innate immune defense against pulmonary infections, but also essential for reducing airway inflammation. For example, SP-D expression increases in response to allergen instillation/inhalation, and that SP-D-deficient mice are hyperreactive to allergen sensitization and challenge (e.g., ovalbumin; OVA) [1]. However, the mechanism by which SP-D reduces allergic/asthmatic conditions and airway inflammation is not clearly understood. We and others have shown that SP-D recognizes several ligands primarily via carbohydrate-recognition domains (CRDs), and enhances their clearance by macrophages [2]. OVA is a glycoprotein that has been extensively used as a model allergen in animal experiments. We hypothesized that SP-D would recognize this protein and enhance its clearance via endocytic/pinocytic pathways of macrophages.

To show the interaction between SP-D and OVA, we first conducted ELISA-based binding assays and found that the CRDs of SP-D recognized OVA and fluorescently labeled DQ OVA (Molecular Probes, OR) via a calcium-dependent (>2.5 $\mathrm{mM} \mathrm{CaCl}_{2}$ ) lectin-carbohydrate interaction. We also tested the biological implications of SP-D-OVA interactions using macrophage RAW 264.7 cells and fluorescence microscopy. To determine the kinetics of OVA uptake and clearance, we incubated RAW cells with DQ OVA (40 $\mu \mathrm{g} / \mathrm{ml})$ in HBSS for various time periods and fixed with ice cold $2 \%$ paraformaldehyde for $30 \mathrm{~min}$. The fluorescent distribution of DQ OVA was analyzed using a Zeiss Axiovert 200M inverted microscope. Internalized and cleaved DQ labeled OVA was detected as bright green spots in RAW cells after a 5 min incubation period (Figure 1A). Since DQ OVA only emits green fluorescence after it is cleaved in lysosomal compartments, we infer that the protein is detected in lysosome related compartments. More DQ OVA signal was detected in the perinuclear regions, cytoplasmic compartments and the cell surface after 15 min of incubation (Figure 1B). The most intense fluorescence was detected in the cytoplasmic compartments after 30 min of incubation (Figure 1B and 1C). Fluorescence emission by DQ OVA was significantly reduced after 45 to 60 min of incubation probably due to further degradations of the fluorescent label (Figure 1D and 1E).

Pre-incubation of DQ OVA (40 $\mu \mathrm{g} / \mathrm{ml})$ with purified human SP-D (hSP-D, $5 \mu \mathrm{g} / \mathrm{ml}$ ), in the presence of 0-5 $\mathrm{mM} \mathrm{CaCl}_{2}$ for $30 \mathrm{~min}$, resulted in a pronounced enhancement of clearance of DQ OVA by RAW cells. This effect was seen when $\mathrm{CaCl}_{2}$ concentration was greater than $3 \mathrm{mM}$ (Figure $2 \mathrm{E}-2 \mathrm{H}$ ). These results were consistent with the ELISA analysis, in which, hSP-D did not bind well to DQ OVA below $2.5 \mathrm{mM} \mathrm{CaCl}_{2}$ concentration. hSP-D was colocalized with fluorescent DQ OVA in some aggregates and inclusions that were localized to the cell surface and the cytoplasmic compartments (Figure 2G; data not shown). In addition, pre-incubating RAW cells (60 min) with SP-D also enhanced the clearance of DQ OVA (e.g., $30 \mathrm{~min}, 37^{\circ} \mathrm{C}$ ) which had similar aggregate features associated with the cell surfaces and the cytoplasmic compartments (Figure 2I-2L).

Taken together, we conclude that interaction between SP-D and OVA requires calcium ions (>2.5-3 $\mathrm{mM}$ ), and the enhancement of clearance occurs, at least, in part by the aggregate formation. Since cells coated with SP-D also show enhanced clearance of DQ OVA and aggregate formation, both pinocytosis and endocytosis may be involved in SP-D-mediated clearance of OVA by macrophages.

References:

[1] B. Schaub et al., Clinc Exp Allergy. 34 (12) (2004) 1815.

[2] N. Palaniyar et al., J Immunol. 174 (11) (2005) 7252. 

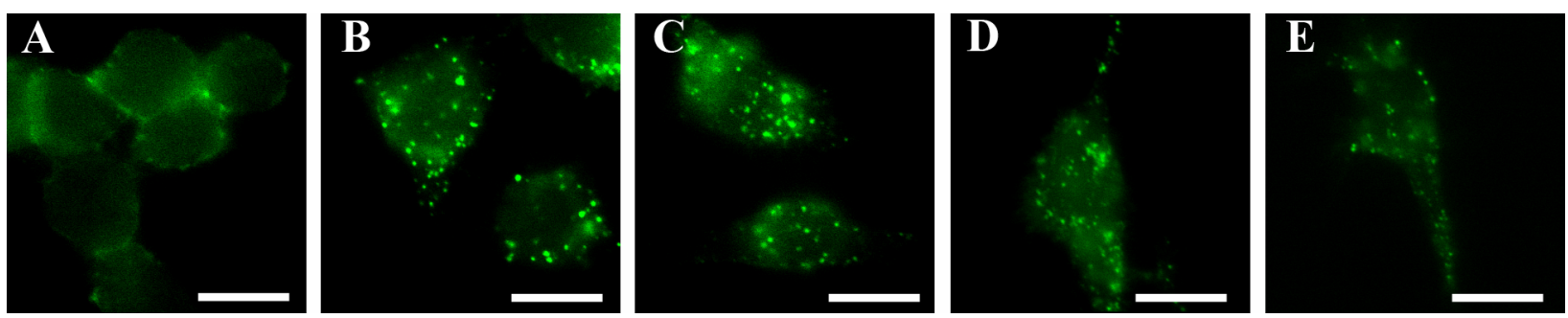

Figure 1. Internalization of DQ OVA. RAW cells were pulsed with DQ OVA (40 $\mu \mathrm{g} / \mathrm{ml})$ in HBSS for $10 \mathrm{~min}$, washed and chased with the same buffer at $37{ }^{\circ} \mathrm{C}$, and fixed with ice cold $2 \%$ paraformaldehyde in HBSS for $30 \mathrm{~min}$. For the 5 min internalization experiment, internalization was immediately stopped on ice after incubation with DQ OVA for $5 \mathrm{~min}$, washed with ice cold buffer to remove free DQ OVA, and fixed with ice cold $2 \%$ pararformaldehyde. Internalized and cleaved DQ OVA was detected by green fluorescence spots. Total internalization time for DQ OVA is (A) 5, (B) 15, (C) 30, (D) 45, and (E) $60 \mathrm{~min}$. Scale bar is $15 \mu \mathrm{m}$.
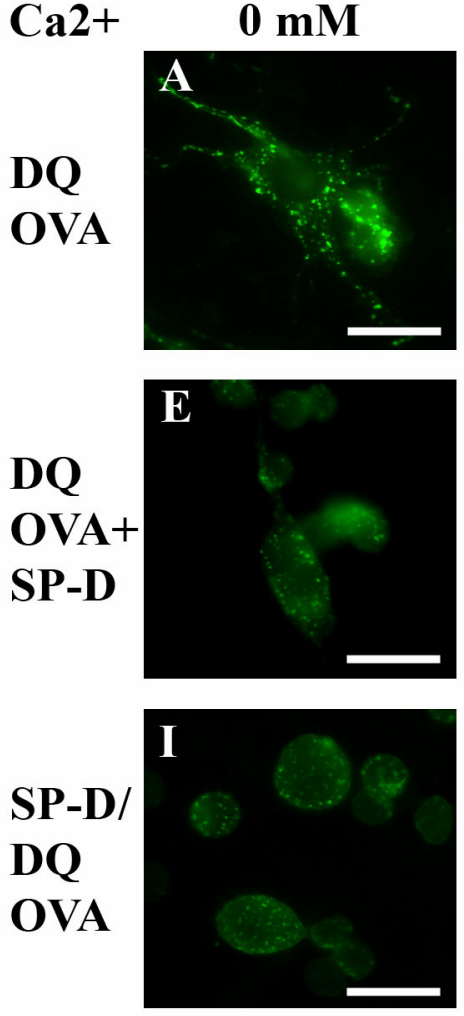

$2 \mathbf{m M}$


$3 \mathbf{m M}$



Figure 2. SP-D enhances the clearance of DQ OVA by macrophage cells. RAW cells were incubated with DQ OVA by the following conditions: DQ OVA $(40 \mu \mathrm{g} / \mathrm{ml})$ in HBSS alone (A-D), DQ OVA $(40 \mu \mathrm{g} / \mathrm{ml})$ and $\mathrm{hSP}-\mathrm{D}(5 \mu \mathrm{g} / \mathrm{ml})$ in HBSS that were already mixed at room temperature for 30 min (DQ OVA+SP-D; E-H), and incubation with hSP-D $(5 \mu \mathrm{g} / \mathrm{ml})$ and DQ OVA $(40 \mu \mathrm{g} / \mathrm{ml})$ separately (SP-D/DQ OVA, I-L), by which RAW cells were incubated with hSP-D $(5 \mu \mathrm{g} / \mathrm{ml})$ in $\mathrm{HBSS}$ at $37^{\circ} \mathrm{C}$ for 60 min followed by DQ OVA incubation. All incubations with DQ OVA were performed at 37 ${ }^{\circ} \mathrm{C}$ for $30 \mathrm{~min}$. RAW cells showed significantly enhanced clearance of DQ OVA after incubation with DQ OVA+SP-D and SP-D/DQ OVA in the presence of $3 \mathrm{mM}$ of $\mathrm{CaCl}_{2}$. Note that DQ OVA aggregation by SP-D was prominent at $\mathrm{CaCl}_{2}$ concentration greater than $3 \mathrm{mM}(\mathrm{G}, \mathrm{K}$, and $\mathrm{L})$, although aggregation may occur at lower calcium concentration (J). Scale bar is $15 \mu \mathrm{m}$. 